\title{
Experimental Demonstration of Active Electromagnetic Cloaking
}

\author{
Michael Selvanayagam and George V. Eleftheriades* \\ The Edward S. Rogers Department of Electrical and Computer Engineering, University of Toronto, Toronto, Canada
}

(Received 1 July 2013; published 12 November 2013)

\begin{abstract}
Active electromagnetic cloaking uses an array of elementary sources to cancel the scattered fields created by an object. An active interior cloak does this by placing the sources along the boundary of the object. This process can be thought of as introducing a discontinuity in the field to cancel out the scattered field by the object. Here, an experimental version of a thin active cloak at microwave frequencies is demonstrated for an aluminum cylinder with a radius of $0.56 \lambda$. The cloak consists of a 12-element magnetic-dipole array. By controlling the weights of the current on each element of the array, the scattering off of the cylinder is reduced in the backward and forward directions. The ability to disguise the aluminum cylinder as another object by varying the weights of the dipole array is also demonstrated. Finally, potential ways of overcoming the constraint of requiring a priori knowledge of the incident field leading to camouflaging-type behavior are discussed.
\end{abstract}

DOI: 10.1103/PhysRevX.3.041011

Subject Areas: Metamaterials

\section{INTRODUCTION}

Cloaking an object from an electromagnetic field represents a major advancement in the ability to control and manipulate electromagnetic radiation. Since the work reported in Ref. [1], the concept of cloaking an object from an incident electromagnetic wave has spurred many different perspectives on how the scattering off of an object can be managed.

In Ref. [1], cloaking is accomplished by covering an object with an anisotropic and inhomogeneous material shell that bends the incident field around the interior volume. This bending allows for any object that fits inside that volume to be hidden, an approach that belongs to the general field of "transformation optics." This rerouting of the incident field comes at the trade-off of requiring extreme material parameters (the permittivity and permeability approaching either zero or infinity). Such an approach is the most general form of material-based cloaking for a monochromatic plane wave, as it can hide any object from any incident field. However, it is not ideal for pulses because of the dispersion inherent in the material parameters and the inherent bending of the fields around the object that is required. As stated in Ref. [2], no material-based electromagnetic cloaks can causally respond to a pulse. Such a metamaterial cloak is also the most difficult to implement and requires approximating the ideal parameters, as well as electrically thick shells that are limited by losses $[3,4]$.

To get around these limitations, cloaking mechanisms have been proposed that use specific knowledge about the

\footnotetext{
*gelefth@ece.utoronto.ca
}

Published by the American Physical Society under the terms of the Creative Commons Attribution 3.0 License. Further distribution of this work must maintain attribution to the author(s) and the published article's title, journal citation, and DOI. object being hidden and the incident field with which it is illuminated. Making assumptions about these quantities allows for cloaks to be tailored to a specific situation. For example, cloaking using transformation optics can be achieved using simpler material parameters if the object is backed by a ground plane [5] or if the cloaking effect is reduced to a known angle of incidence [6,7].

Plasmonic cloaking [8] uses the size of the object and its material composition to tailor the scattering of the object. By assuming an electrically small object, the scattering can be described by its lowest-order scattering term (the monopole or dipole term, depending on the object), which gives rise to a cloak made up of a homogeneous plasmonic shell (permittivity $<0$ ) $[9,10]$. This shell can also be reduced to a low-profile surface described by an inductive impedance [11].

Another form of cloaking designed for the microwavefrequency range is tailored for cylindrical objects of various dimensions using parallel metal sheets that guide the waves around or through the object being hidden $[12,13]$.

The examples given above are all passive ways of cloaking an object using materials or surfaces. Another way of accomplishing a cloaking effect for waves is to use an active approach using sources instead of materials. Active cloaking has previously been proposed for acoustic waves [2,14]. As discussed in Ref. [2], sources placed around an object could be used to hide an object from pulses by using prior knowledge about the field to determine the weight on each source (predetermined cloaking). A more sophisticated form of cloaking was also discussed in Ref. [2] (true cloaking), which could be achieved by constantly sampling the field to set the weights in real time. Such a method, however, requires nonlocal knowledge about the field at each source or sensor. While this is not possible for electromagnetic cloaks, it is possible for other waves (such as acoustic waves) that allow for information to flow faster than the wave itself. Active cloaking with 
sources for electromagnetic waves was briefly described in Ref. [2], where it was stated that predetermined cloaking is possible, while true cloaking is not feasible because of causality constraints.

For active cloaking of electromagnetic fields, the sources could be antennas with specific radiation patterns. Typically, arrays of antennas are used in communication links or radar detection. Here, for active cloaking, the antennas are used instead to tailor the scattering of the object being cloaked. This method also brings to mind techniques such as active radar jamming [15], which uses noise and signal processing to alter the radar profile of a target. We note that these approaches do not necessarily cancel the scattered fields surrounding an object but disguise or mask an object along certain directions of interest. On the other hand, active cloaking in the context of this paper seeks to alter the scattered fields (including their total cancellation) at all directions in space, which is a more general problem. The basic form of active cloaking for electromagnetic fields assumes a predetermined field and falls into two main classes, exterior and interior methods.

Exterior methods [16-18] require an array of multipole sources (at least three) that create a region of space where the field is zero. The weights and order of the multipole terms of these sources are determined by the properties of the incident field and the size of the space where we desire the field to be zero. Through interference, these sources, along with the incident field, create the zero-field region. Because the sources are far away from the location of the object, any object can be placed in this zero-field region without disturbing the interference of the multipole sources and the incident field. This process allows for no scattering off of the object and thus no scattered field. It can be seen that exterior methods do not require any information about the scatterer itself, as the goal is to create a zero-field region only, provided that the sources are far away from the scatterer.

Interior methods place sources around the object itself to cancel out the scattered fields of the objects. Because interior methods place sources very close to the scatterer, they have to be designed with the properties of the scatterer in mind. These sources are Huygens sources (orthogonal electric and magnetic dipoles), and the weights of the source are then ultimately determined by the incident field and the scatterer. By superimposing these sources with the incident field, the object is hidden. As mentioned, Ref. [2] discusses interior cloaking, while another equivalent way of formulating the problem, specific to the electromagnetic case, using the equivalence principle (or discontinuous fields) was given in Refs. [19,20].

While passive material cloaks have been demonstrated before, there has been no demonstration of active cloaking for electromagnetic fields in the literature. In this work, an initial prototype of an active cloak is demonstrated, specifically, an active interior cloak for a predetermined incident field. In the following section, the synthesis of the active cloak is briefly reviewed. Then, the design and measurement of the prototype cloak is discussed, followed by an analysis of the measured results. Finally, ways to estimate the incident field using the cloak itself are discussed.

\section{CONCEPT}

In Ref. [19], the problem of active electromagnetic cloaking was formulated using the equivalence principle $[21,22]$, as opposed to the equivalent formulation in Ref. [2]. Specifically, this was achieved by imposing appropriate orthogonal electric and magnetic currents (Huygens sources) around a scatterer. In Ref. [20], this concept was extended for various other cloaking scenarios (e.g., leaving the interior fields unchanged or for modifying the scattered fields) and for general wave-front manipulation by a metasurface. To briefly review this cloaking scheme, the two-dimensional geometry pictured in Fig. 1 is used, where a steady-state point source, with a frequency $\omega$ in the microwave spectrum (assuming a $e^{j \omega t}$ convention), is incident on a cylindrical scatterer. The cylinder is of radius $r=a$ and made out of a material with an arbitrary dielectric constant $\varepsilon$. Note that this two-dimensional scenario can easily be extended to three dimensions. The electric field of the incident plane wave is polarized perpendicularly to the $x y$ plane, as shown in Fig. 1, and will be the assumed polarization throughout this work. The total electric field outside the cylinder, $\mathbf{E}_{\mathbf{t}}$, in such a scenario is a superposition of the incident field and the scattered field, given by

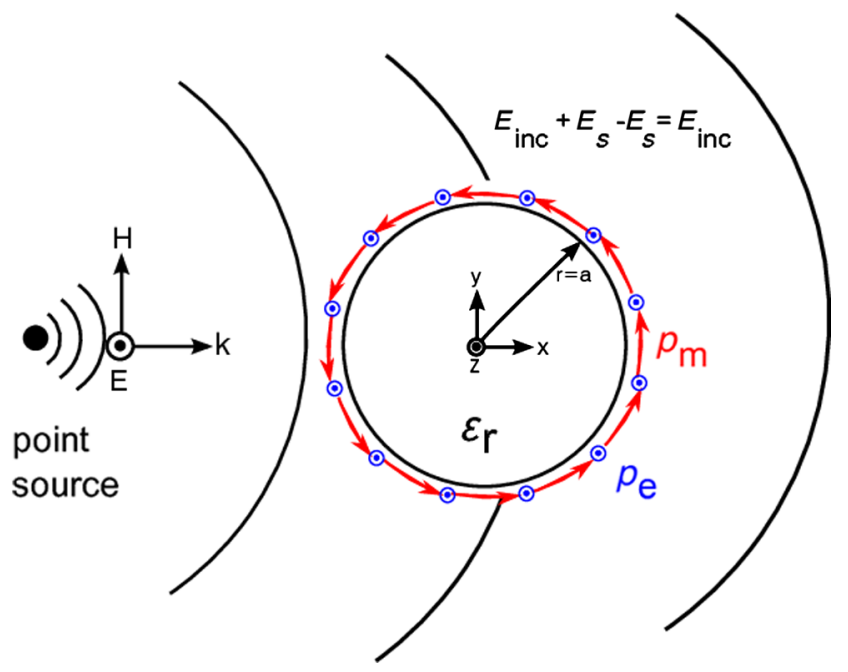

FIG. 1. The geometry of the scattering problem discussed. A point source with the illustrated polarization illuminates a circular PEC scatterer. To cloak the PEC scatterer, it is surrounded by magnetic dipoles, with a specific weight on each dipole determined by the scattered field. 


$$
\begin{aligned}
\mathbf{E}_{\text {total }}= & E_{\text {total }} \hat{\mathbf{z}}=\left(E_{\mathrm{inc}}+E_{s}\right) \hat{\mathbf{z}} \\
= & E_{o} e^{j \theta} H_{o}^{(2)}\left(k_{o}\left|\rho-\rho^{\prime}\right|\right) \hat{\mathbf{z}} \\
& +E_{o} e^{j \theta} \sum_{n=-\infty}^{n=\infty} A_{n} H_{n}^{(2)}\left(k_{o} \rho^{\prime}\right) H_{n}^{(2)}\left(k_{o} \rho\right) e^{j n\left(\varphi-\varphi^{\prime}\right)} \hat{\mathbf{z}},
\end{aligned}
$$

where $\rho^{\prime}$ and $\varphi^{\prime}$ describe the location of the source, $E_{o} e^{j \theta}$ is the complex weight of that source, and $H_{n}^{(2)}$ is the Hankel function of the second kind. Here also, the scattered field is decomposed into cylindrical wave functions, and $A_{n}$ are the coefficients for each term of the sum of cylindrical wave functions. These are determined by imposing the continuity of both the tangential electric and magnetic fields at the boundary.

To cloak this object, sources can be superimposed on its boundary, and these sources must radiate a field that cancels out the scattered field, leaving the incident field only. To determine the properties of these sources, their function can be interpreted as imposing a discontinuity in the electromagnetic field at the boundary. By this, we mean that since the tangential incident and scattered electromagnetic fields outside the cylinder are continuous with the fields inside the cylinder across the boundary of the object, any other field existing either inside or outside the cylinder would create a discontinuity. This discontinuity must be supported by corresponding electric and magnetic currents [20,23].

Thus, in the case of a cloak where one would like to cancel the scattered field outside the cylinder, these electric and magnetic currents are given by the negative of the tangential scattered field at the surface of the cylinder,

$$
\begin{gathered}
\mathbf{M}_{\mathrm{s}}=\hat{\mathbf{n}} \times \mathbf{E}_{\mathbf{s}}, \\
\mathbf{J}_{\mathrm{s}}=-\hat{\mathbf{n}} \times \mathbf{H}_{\mathrm{s}},
\end{gathered}
$$

where $\hat{\mathbf{n}}$ is the outward normal of the surface. These continuous electric and magnetic currents can then be approximated by an array of discrete electric and magnetic dipoles. The weights of these dipoles are given by

$$
\begin{gathered}
\mathbf{p}_{\mathbf{m}}=\frac{\mathbf{M}_{\mathbf{s}} a d \varphi d z}{j \omega}, \\
\mathbf{p}_{\mathbf{e}}=\frac{\mathbf{J}_{\mathbf{s}} a d \varphi d z}{j \omega},
\end{gathered}
$$

where $a d \varphi$ and $d z$ give the discrete physical size of each dipole. The minimum number of required dipoles is determined by the Nyquist criterion to effectively capture the fastest variation in the electric and magnetic currents around the boundary of the object. For the polarization in question here, the magnetic dipoles lie in the plane, and the electric dipoles lie perpendicular to the plane.

Of course, as discussed above, the weights for all of these dipoles are determined by the scattered field, which itself is determined by the incident field. Thus, either a priori knowledge of the incident field is required or, as will be discussed later, the incident field has to be estimated.

\section{A. Cloaking a perfect electric conductor cylinder}

From the previously discussed theory, the experimental realization of cloaking in this paper will be demonstrated for a perfect electric conductor (PEC) cylinder. For this scenario, the cloak will be designed for the microwavefrequency range, with a design frequency around $1.5 \mathrm{GHz}$, and the size of the PEC cylinder is given by a radius of $a=0.56 \lambda$. Note that this is an appreciably large object with respect to the incident wavelength. The PEC scatterer is illuminated by a monopolar point source $3 \lambda$ away from the object. Because the object is a perfectly conducting cylinder, the scattered fields of the cylinder are described by enforcing the continuity of the electric fields only (as opposed to a dielectric cylinder, where both the electric and magnetic fields are continuous across the boundary). Then, we only need Eq. (2) to describe the scattered fields, as per the equivalence principle [23], and thus the desired discontinuity, which is the negative of the scattered fields. Using Eq. (2), the required magnetic currents to impose a discontinuity in the field are then given by the following expressions,

$$
\mathbf{M}_{\mathbf{s}, \mathbf{c l o a k}}=E_{o} e^{j \theta} \sum_{n=-\infty}^{n=\infty} A_{n} H_{n}^{(2)}\left(k_{o} \rho^{\prime}\right) H_{n}^{(2)}\left(k_{o} a\right) e^{j n\left(\varphi-\varphi^{\prime}\right)} \hat{\boldsymbol{\varphi}}
$$

where the scattering coefficients are given by $A_{n}=$ $-J_{n}(k a) / H_{n}^{(2)}(k a)$. The magnetic-current distributions are plotted in Fig. 2. With the magnetic currents on the
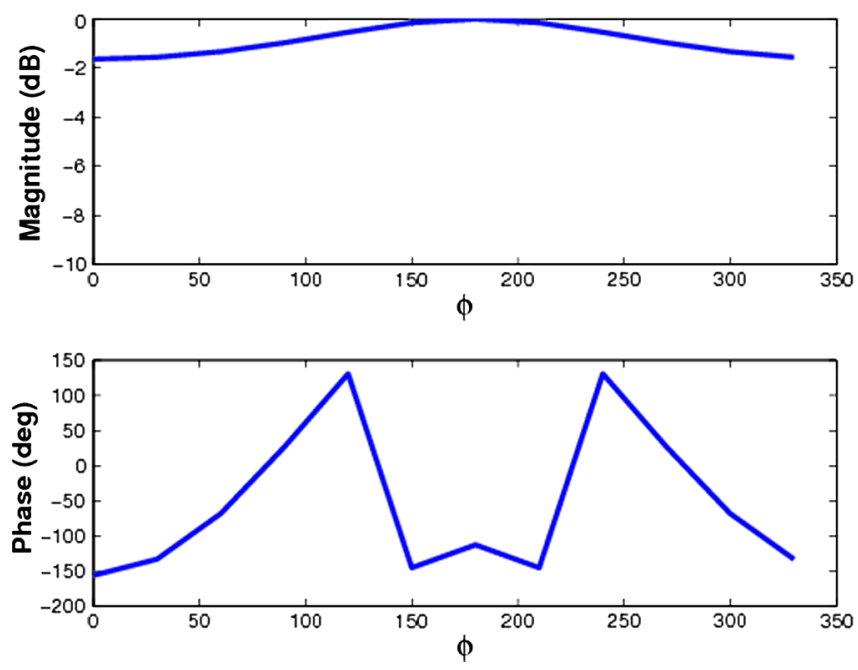

FIG. 2. The magnitude and phase of the magnetic currents required to cloak a PEC cylinder. The magnetic currents are determined by the scattered field at the boundary of the PEC cylinder. 
boundary of the PEC scatterer defined, this current distribution can be constructed using a magnetic-dipole array, which will be discussed in the following section.

\section{DESIGN AND MEASUREMENT}

To demonstrate this form of active cloaking, the cloak will be constructed from an array of elementary dipole antennas, as stated earlier. The measurement setup of the cloak needs to be considered as well. Similarly to other experimental demonstrations of cloaking and lensing $[9,24,25]$, our cloak will be situated in a parallel-plate waveguide that allows for the emulation of an environment with an infinite extent along the $z$ axis. To discuss these details, a brief overview of the measurement setup is given, followed by some details regarding the design of the cloak.

\section{A. Measurement setup}

Our measurement setup is designed for the microwavefrequency range with an operating frequency around $1.5 \mathrm{GHz}$ and is illustrated in Fig. 3, along with the corresponding coordinate system. The waveguide is fabricated from two rectangular steel sheets that are held parallel to each other via plastic spacers around the perimeter of the waveguide. The sheets are $1626 \mathrm{~mm} \times 1092 \mathrm{~mm}$. The height of the waveguide is $38 \mathrm{~mm}$, which allows for the waveguide to support only a TEM mode, as all other modes are cut off. On the periphery of the waveguide, foam absorbers (Emerson-Cummings, AN-77) are placed to terminate the guide and reduce reflections from its ends.

As shown in Fig. 3, a small monopole antenna is placed at one end of the waveguide to excite the waveguide itself. This is a point-source-like excitation, and it gives rise to cylindrical wave fronts in the waveguide. The amplitude and phase of the current on the monopole are controlled through a voltage-controlled phase shifter (described below in the following section) in series with a voltagecontrolled attenuator (Mini-circuits ZX73-2500-S+). The

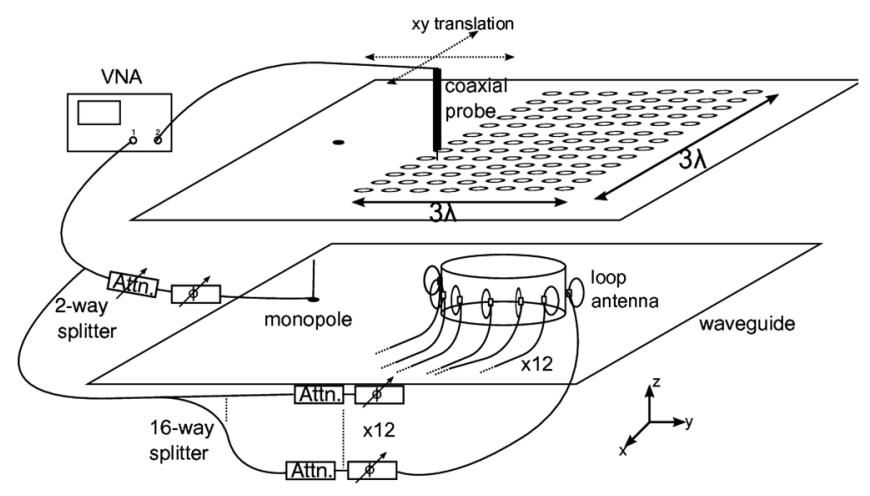

FIG. 3. A schematic of the measurement setup used to measure scattering and cloaking off of an aluminum cylinder. Note that the waveguide plates sit flush against the aluminum cylinder. Also note that absorbers are used to close off the waveguide (not shown). purpose of the attenuator and phase shifter is to control the magnitude and phase of the source, such that the source is synchronized with the active cloak itself, as the incident field must be known ahead of time.

The scatterer in the setup is an aluminum cylinder also placed in the waveguide at $3 \lambda$ away from the monopole antenna (600 mm, center to center), which approximates a PEC scatterer. The aluminum cylinder has a height of $38 \mathrm{~mm}$ and a radius of $112 \mathrm{~mm}$.

To measure the fields inside the waveguide, the top plate of the waveguide is perforated with small subwavelength circular holes with a radius of $5 \mathrm{~mm}$. These holes are repeated in a square grid with a period of $15 \mathrm{~mm}$. Because the holes are subwavelength, they have a minimal perturbation on the fields inside the waveguide, as confirmed through full-wave simulation. To probe the fields, a small semirigid coaxial cable is used, with its center conductor exposed. This cable picks up the electric field in the waveguide $\left(E_{z}\right.$ in the given coordinate system) and is placed on a translation stage that moves the probe into and out of each hole at a predetermined height. The range of motion of the translator allows for an area of $600 \mathrm{~mm}$ by $600 \mathrm{~mm}$ to be scanned or, in terms of wavelengths, $3 \lambda \times 3 \lambda$, as shown in Fig. 3. The measurements are recorded using a Vector Network Analyzer (VNA, Agilent E8364B) with port 1 of the VNA connected to the monopole antenna and port 2 connected to the probe. The measured $S_{21}$ (transmission from port 1 to port 2) at each position yields a relative map of the complex electric field $\left(E_{z}\right)$ as a function of space. These field plots are rescaled to fit in a -1 to 1 scale. Note that for the field plots, it is not the absolute value that is important but the relative values in space which map out the wave behavior in the waveguide.

To demonstrate this measurement setup, the field plot of the monopole source scattering off of the aluminum cylinder is shown in Fig. 4(a), with the real part of the field plotted. Here, the distinctive shadow region created by the object is seen in the field plot (as well as the scattering in the other directions). This case has good agreement with the ideal PEC case shown in Fig. 4(b) (found using COMSOL multiphysics).

\section{B. Designing the cloak}

The continuous magnetic currents required to form the active cloak can be approximated by a discrete array of sources, as stated earlier. As shown in Fig. 2, the magnetic currents required for the various cloaking schemes, in general, vary in both amplitude and phase. Thus, to effectively implement the cloak, a discrete source, and a way of controlling the weight of that source, is required for both its magnitude and phase. The simplest discrete source to model a magnetic current is a magnetic dipole, which from antenna theory, takes on the form of an electrically small loop antenna (a loop with radius $r<\lambda / 10$ ) [23]. The 


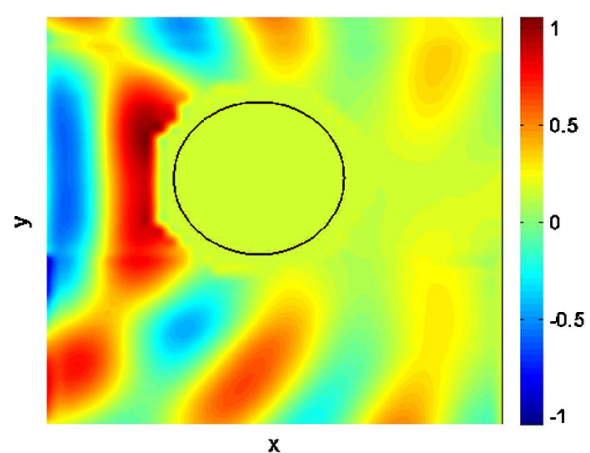

(a)

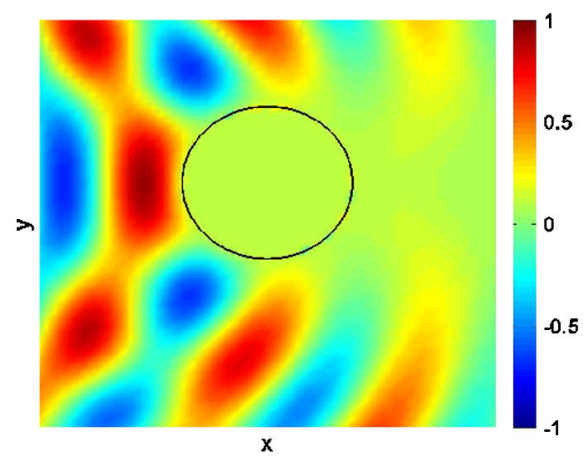

(b)

FIG. 4. (a) The real part of the total measured electric field for a point source scattering off of an aluminum cylinder. The inner circle shows the boundary of the aluminum cylinder. (b) The real part of the total electric field for a point source scattering off of a PEC cylinder, as found using COMSOL multiphysics.

relationship between the current on the loop to the effective magnetic-dipole moment is proportional and given by $I=$ $\left|\mathbf{p}_{\mathbf{m}}\right| /(\mu S)$, where $S$ is the area of the loop, with the dipole moment perpendicular to the face of the loop [23]. To construct these antennas, printed loops are fabricated on FR-4 circuit boards with a radius of $5 \mathrm{~mm}$. These antennas are designed for a frequency around $1.5 \mathrm{GHz}$ and are pictured in Fig. 5(a). Also seen in Fig. 5(a) is the feed for the antenna itself. To confirm the dipolelike properties of the antenna, the measured radiation pattern is shown in Fig. 5(b), where the two-lobe dipolar pattern is clearly visible.

To determine the number of antennas that are needed, the magnetic-current distribution in Fig. 2 is used. To effectively represent this distribution with an array of discrete dipoles, we need to apply sampling theory to determine the number of dipoles. Taking the Fourier transform numerically (FFT) of Fig. 2, we find that a minimum of 12 dipoles is required to effectively sample the current shown in Fig. 2.

With the number of dipoles given, the magnitude and phase for the current on each element of the 12-dipole array need to be set. To do this, custom-designed phase shifters modeled after Ref. [26] are used to control the phase on the antenna and off-the-shelf SMA attenuators are

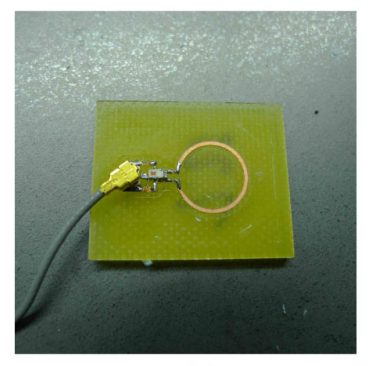

(a)

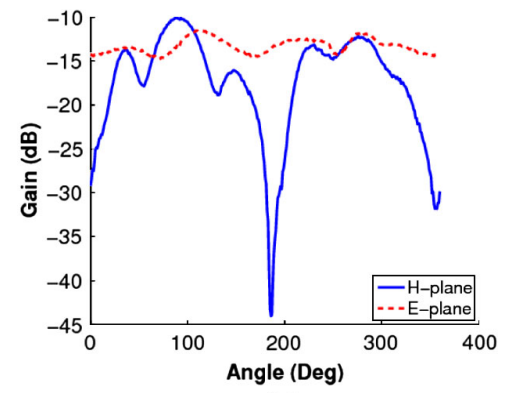

(b)
FIG. 5. (a) The fabricated loop antenna. (b) The measured radiation pattern of the loop antenna. Two cuts of the 3D pattern are shown, indicating the dipolar nature of the antenna. used to alter the magnitude. The phase shifters are printed on a Rogers 3003 circuit board using varactor diodes (Aeroflex-MGV125-26-E28X), with each phase shifter controlled by a single (external) voltage source. By varying the voltage between 0 and $10 \mathrm{~V}$ the phase shifter can cover over a $360^{\circ}$ phase range, thus allowing for the proper phasing of the current on each loop. For the cloak, because the amplitude of the magnetic current varies very approximately by $\pm 1 \mathrm{~dB}$ across the cylindrical boundary, as shown in Fig. 2, the magnetic current can be approximated with a constant amplitude, without the need for attenuators. This is essentially a far-field approximation, where the driving point source is assumed to radiate a plane wave at the boundary of the cylinder, as a plane wave would have a uniform amplitude over the cylinder. However, we demonstrate in the following sections that amplitude control is useful for other magnetic-current distributions. In those scenarios, attenuators are used that have a fixed attenuation in the $-1 \mathrm{~dB}$ to $-20 \mathrm{~dB}$ range (Crystek Corporation). For the required magnitude, the appropriate attenuator is used.

The complete setup is pictured in Fig. 6, with the loop antennas placed next to the cylindrical scatterer in foam

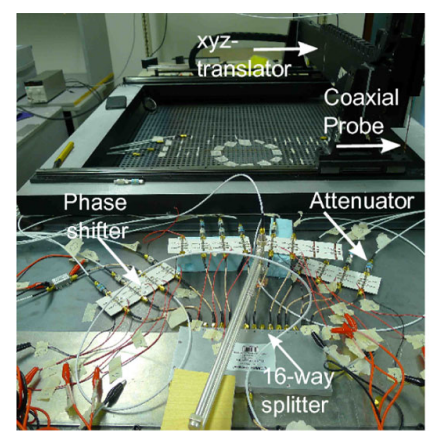

(a)

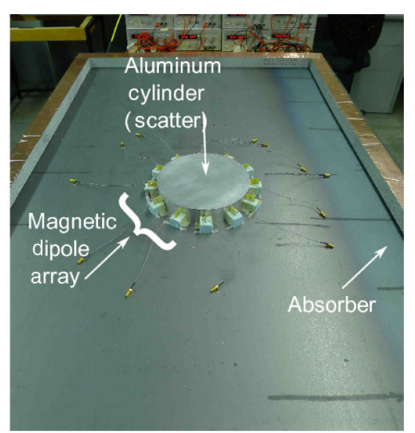

(b)
FIG. 6. (a) The exterior of the waveguide setup. Pictured are the phase shifter, translator, and coaxial probe. The VNA is just outside of the picture and to the left. (b) The interior of the waveguide setup. The loop antennas conformally surround the scatterer. 
stands. The phase shifters and attenuators are placed outside of the waveguide, as shown in Fig. 6(a), because of the discrete nature of the setup. It is important to emphasize that a more compact design could be envisioned in the microwave regime using an integrated circuit approach [27].

To feed the dipole array, port 1 of the VNA is split through a two-way power splitter (Mini-Circuits ZAPD2-21-3W-S+), with the first feed going to the monopole antenna and the second feed to the dipole array. This second feed is then passed through another 16-way power splitter (Mini-Circuits ZC16PD-24+) to feed each of the 12 phase-shifters-attenuators-loop antenna blocks (with the remaining ports terminated).

To demonstrate the cloaking effect, the voltages on each phase shifter are set to generate the desired phase. For the monopole antenna, the phase shifter and attenuator are tuned to ensure that the monopole antenna and loop antenna arrays are synchronized.

\section{MEASURED RESULTS}

With the complete description of the experimental setup, the performance of this cloak can now be examined. Before looking at the full cloaking effect, the capabilities of the magnetic-dipole array in the presence of the aluminum cylinder are discussed.

If the magnetic-dipole array is properly phased in the presence of the aluminum cylinder, a field distribution that resembles the scattered field of the cylinder illuminated by a point source is expected. In Fig. 7(a), the real part of the measured field generated by the magnetic-dipole array is shown. Also shown are the scattered fields for the ideal PEC cylinder found from full-wave simulation and the scattered field found from the measured total field of the aluminum cylinder by itself. The latter is determined by subtracting the measured total field from the measured incident field (the field in the empty waveguide). As shown in Fig. 7, good agreement is observed in all three scenarios. There are some discrepancies and variations in the amplitude of the measured fields. These differences are due to inherent limitations in the cloak itself caused by amplitude and phase variations in the loop antennas and phase shifters. These limitations arise from the fabrication tolerances and variations in these devices and in the measurement setup itself. Overall, one can see that if the monopole and magnetic-dipole arrays are properly phased, the scattered field could be reduced.

With this confirmation of the capability of the magneticdipole array, one can now align the phase and amplitude of

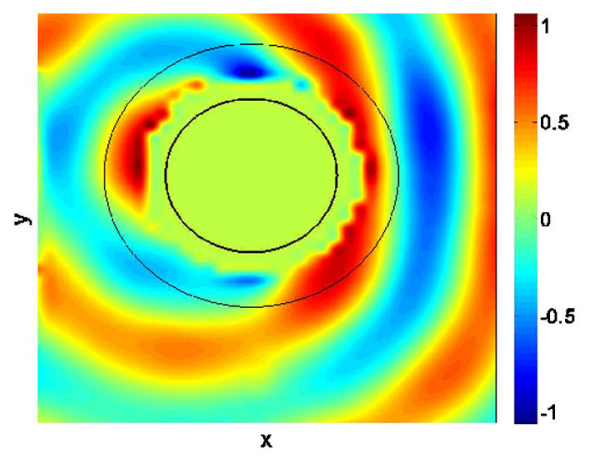

(a)

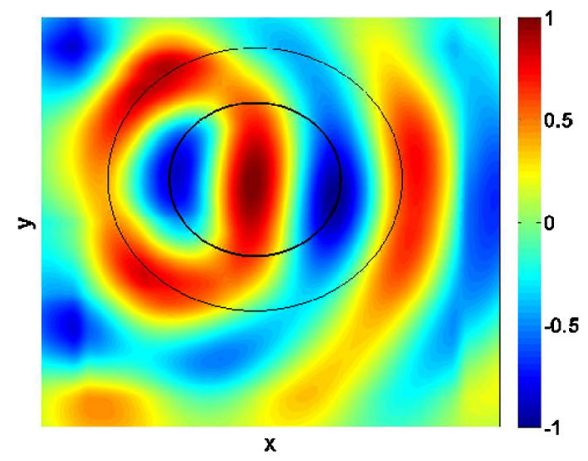

(b)

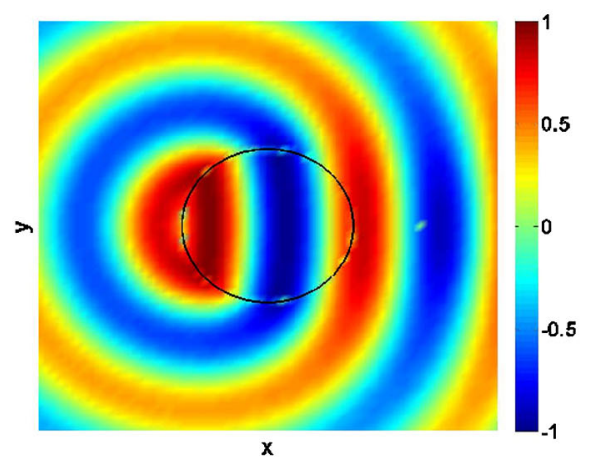

(c)

FIG. 7. (a) The measured real part of the electric field created by the magnetic-dipole array alone in the presence of the aluminum cylinder. The larger circle is the circle on which the near-field data are used to calculate the far-field patterns. (b) The measured scattered field of the aluminum cylinder found by subtracting the total field shown in Fig. 4(a) from the measured incident field (not shown). The larger circle is the circle on which the near-field data are used to calculate the far-field patterns. (c) The simulated real part of the scattered electric field of the cloak computed using COMSOL. 


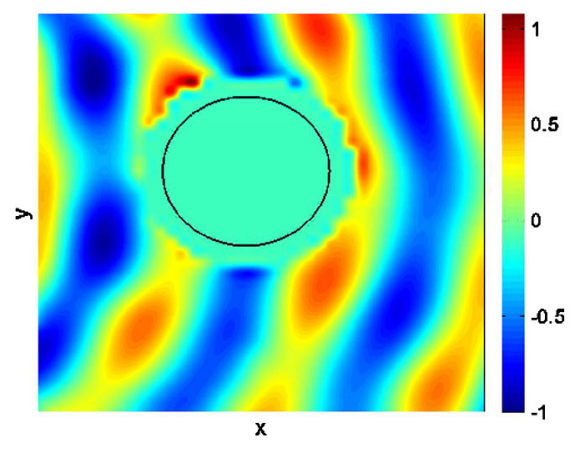

(a)

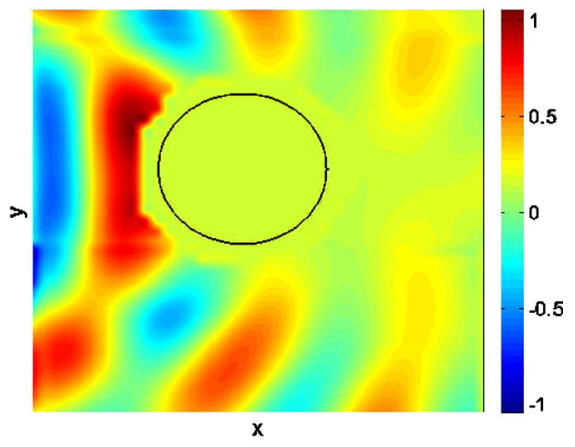

(b)

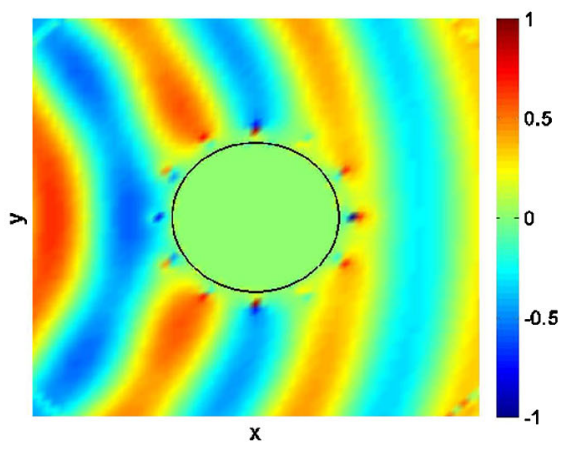

(c)

FIG. 8. (a) The measured real part of the total electric field created by the magnetic-dipole array and the incident field from the monopole antenna in the presence of the aluminum cylinder. Note the shadow region has disappeared, and cylindrical wave fronts have been restored. (b) The measured total electric field for the scattering off of the bare aluminum cylinder, replotted again from (a). Note the shadow region. (c) The simulated cloak using ideal magnetic dipoles, found using COMSOL.

the monopole source with those of the magnetic-dipole array to demonstrate the behavior of the cloak. The measured field in this scenario is plotted in Fig. 8(a), where in comparison to Fig. 4(a), the wave fronts surrounding the aluminum cylinder are restored to the state without the scatterer present, which in this case corresponds to cylindrical-like wave fronts. This case can be especially observed when comparing the shadow region for the scattering off of the PEC cylinder, which is plotted again in Fig. 8(b), to the lack of a shadow in Fig. 8(a). For comparison, the simulated behavior of the cloak using COMSOL multiphysics is also shown in Fig. 8(c), with a good agreement between the two as well. Here, the discrepancies between the simulated and measured results are caused by the amplitude and phase variations in the loop antennas and phase shifters mentioned above, which make up each array. This situation can be observed in the approximate $1 / \sqrt{\rho}$ decay seen in the simulated results, which is not present in the measured results.

\section{A. Far-field plots}

To further characterize the cloak, one can take the measured fields and find the bistatic cross section (BCS) of the bare aluminum cylinder compared to the cloak itself. For a two-dimensional configuration, which is what the parallel-plate waveguide environment emulates, the BCS is defined as $\sigma(\theta)=\lim _{\rho \rightarrow \infty} 2 \pi \rho \frac{E_{s}}{E_{i}}$ [28]. However, this definition assumes knowledge of the far field, whereas for this measurement setup, the near field of the scatterer is being measured. Instead, given that near-field measurements are available, the scattered field determined from the measured data is projected onto cylindrical harmonics, thus enabling the computation of the far field [29].

This process is carried out by finding the measured scattered field on a circle concentric with the scatterer itself, with radius $\rho=b$, as shown in Fig. 7(b). Again, the scattered field is determined by subtracting the measured incident field in the empty waveguide from the measured field in the presence of the scatterer. Following Ref. [29], this scattered field is expanded in terms of cylindrical harmonics, assuming a point-source illumination, which gives

$$
E_{s z}=\sum_{n=-\infty}^{n=\infty} a_{n} H_{n}^{(2)}\left(k_{o} \rho_{o}\right) H_{n}^{(2)}\left(k_{o} b\right) e^{j n(\varphi-\pi)},
$$

with $a_{n}$ the coefficients for the expansion and $\rho_{o}$ the distance between the scatterer and the source. The scattering coefficients are found by treating the above as a Fourier series, which gives

$$
a_{n}=\frac{1}{2 \pi H_{n}^{(2)}\left(k_{o} b\right) H_{n}^{(2)}\left(k_{o} \rho_{o}\right)} \int_{o}^{2 \pi} E_{s} \exp (-j n \varphi) d \varphi
$$


Finally, the BCS in the far field is given by

$$
\sigma(\varphi)=\left|\sum_{n=-\infty}^{n=\infty} \exp (j n \varphi) a_{n}\right|
$$

Before presenting the measured results, a couple of limitations with this calculation using the measured data are noted. Given the measurement setup, there are limitations due to the quality of the absorbers (approximately $-20 \mathrm{~dB}$ of attenuation), the sensitivity of the probe [29], and finally, the directivity of the scattered field itself, which, for the given scatterer, is not a very directive pattern. With these limitations, there are constraints on the accuracy at which one can extract the BCS data. Thus, for the cloak, the BCS data are used to simply examine the relative change between the uncloaked cylinder and the cloaked cylinder in order to get an estimate of how well the cloak performs.

With this in mind, the BCS patterns for the bare aluminum cylinder and the cloak are presented in Fig. 9. For comparison, the BCS of the magnetic-dipole array only, in the presence of the cylinder, is also plotted, along with the theoretical calculation for a point source scattering off of a bare PEC cylinder. Note that the measured field data used to calculate the BCS values were first normalized with respect to the incident field measured in the waveguide. Comparing, at first, the measured BCS for the bare aluminum cylinder to the theoretical value, we find that the general shape is tracked by the measured data, with an increase in the scattering in the forward direction due to the shadow created by the scatterer. However, there are variations in the measured data along the backscattering directions. There is also an overall agreement between these two plots with the BCS of the magnetic-dipole array only.
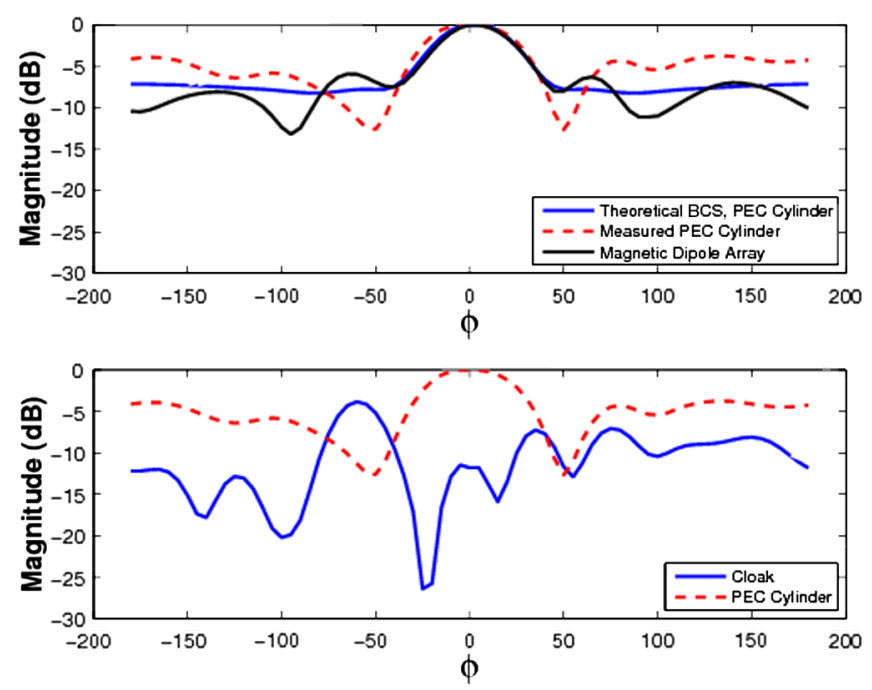

FIG. 9. The calculated BCS patterns for various scenarios. The upper plot shows the measured BCS for the bare aluminum cylinder, the magnetic-dipole array, and the theoretical BCS for a PEC cylinder. The lower plot shows the measured BCS for the bare aluminum cylinder and the active cloak.
Nonetheless, as stated earlier, these data are used to compare to the cloaked BCS, which is also shown in Fig. 9, and a dramatic drop in the BCS is seen. Relatively speaking, in both the forward and backward directions, drops of $-11 \mathrm{~dB}$ and $-8 \mathrm{~dB}$ are observed, respectively. Overall, the cloak suppresses the scattering of the object across most angles, though it is noticed that at $-55^{\circ}$ the scattering increases slightly. This result can be seen in the field plot, as well where an increased intensity in the field is present. As stated above, such an effect is due to the overall variation in the fabricated antennas and phase shifters. Using the BCS data, we can find the scattering width by summing up the scattering coefficients $a_{n}$. Again, we use this metric to simply evaluate the reduction in scattering given by the cloak. Between the two cases of the bare cylinder and the cloaked cylinder, the total scattering width drops from $0.403 \mathrm{~m}$ for the bare cylinder to $0.133 \mathrm{~m}$ for the cloaked cylinder. Overall, this situation indicates that the active cloak is effective at reducing the scattering of an object for a known incident wave.

\section{DISGUISING A CYLINDER}

Because the weights of the magnetic-dipole array needed to cancel the scattered field are purposefully set, other configurations of magnetic currents are conceivable, which can create interesting field distributions. Here, the weights of these dipoles will be configured to impose a discontinuity in the field, which causes the cylinder to appear as another object [20]. We note that similar demonstrations can be done for active exterior cloaks as well $[17,18]$. To accomplish this, the fields scattered by the object must continue to be canceled, but on top of this cancellation, the field required to cause the scatterer to look like a different object must be superimposed. From this, the equivalent magnetic currents are given by

$$
\mathbf{M}_{\mathbf{s}}=\hat{\mathbf{n}} \times\left(\mathbf{E}_{\mathbf{s}}-\mathbf{E}_{\mathbf{d}}\right),
$$

where $E_{d}$ are the fields that cause the cylinder to look like another object. Here, two "disguises" are demonstrated. The first disguise makes a PEC cylinder look like a smaller PEC cylinder with radius $a_{d}=0.2 \lambda$. The second disguise makes a PEC cylinder look like a dielectric cylinder with $\varepsilon_{r}=10$ and radius $a_{d}=0.4 \lambda$.

To disguise a PEC cylinder as a different cylinder, the discontinuity in the field is given by superimposing the negative part of the scattered field with the desired disguise. This process gives a magnetic-current distribution of

$$
\begin{aligned}
\mathbf{M}_{\mathbf{s}, \mathbf{d}}= & E_{o} e^{j \theta}\left[\sum_{n=-\infty}^{n=\infty} A_{n} H_{n}^{(2)}\left(k_{o} \rho^{\prime}\right) H_{n}^{(2)}\left(k_{o} a\right) e^{j n\left(\varphi-\varphi^{\prime}\right)}\right. \\
& \left.-\sum_{n=-\infty}^{n=\infty} B_{n} H_{n}^{(2)}\left(k_{o} \rho^{\prime}\right) H_{n}^{(2)}\left(k_{o} a_{d}\right) e^{j n\left(\varphi-\varphi^{\prime}\right)}\right] \hat{\boldsymbol{\varphi}},
\end{aligned}
$$

where $B_{n}$ are the coefficients for the scattered field for the desired disguise. To make a PEC cylinder look like a smaller PEC cylinder, the coefficients $B_{n}$ are given by 

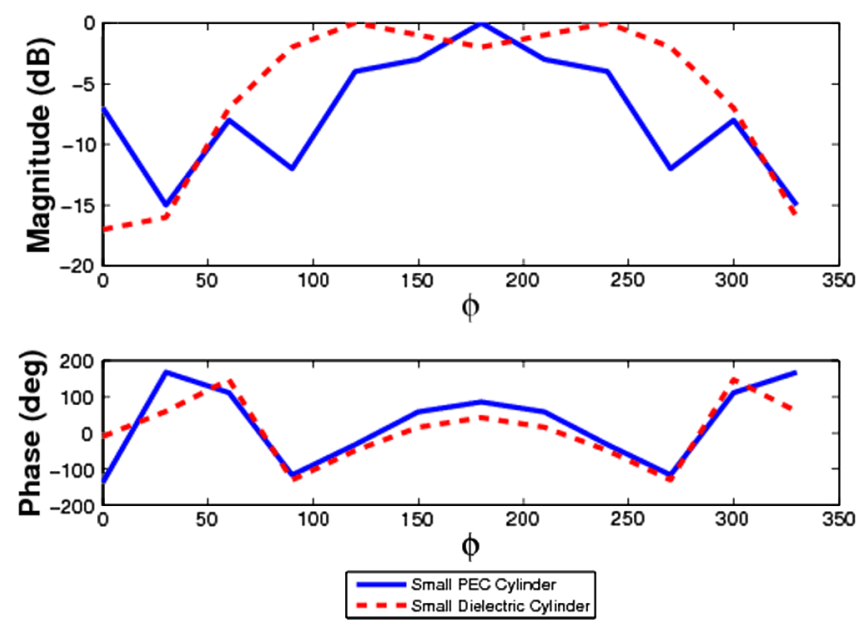

FIG. 10. The magnitude and phase of the magnetic currents required to disguise a PEC cylinder. The blue curves show the required magnetic currents for disguising the PEC cylinder as a smaller PEC cylinder. The red curves show the required magnetic currents for disguising a PEC cylinder as a smaller dielectric cylinder.

$$
B_{n}=\frac{-J_{n}\left(k_{o} a_{d}\right)}{H_{n}^{(2)}\left(k_{o} a_{d}\right)}
$$

Similarly, to make a PEC cylinder look like a dielectric cylinder, the coefficients for the scattered field of a dielectric cylinder are given by

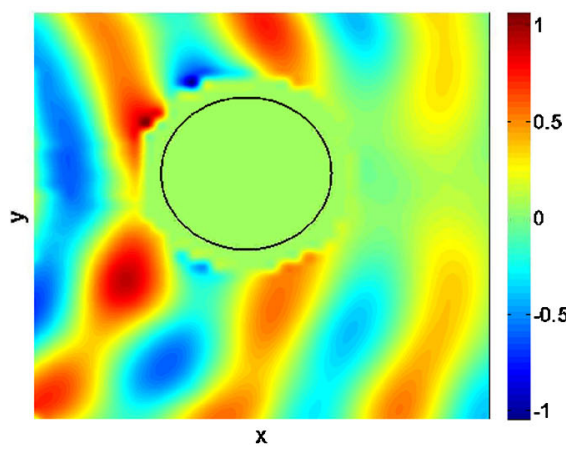

(a)
$B_{n}=-\frac{k_{1} \varepsilon_{r} k_{o}^{2} J_{n}^{\prime}\left(k_{1} a_{d}\right) J_{n}\left(k_{o} a_{d}\right)+k_{1}^{2} k_{o} J_{n}\left(k_{1} a_{d}\right) J_{n}^{\prime}\left(k_{o} a_{d}\right)}{k_{o}^{2} k_{1} \varepsilon_{r} H_{n}^{(2)}\left(k_{o} a_{d}\right) J_{n}^{\prime}\left(k_{1} a_{d}\right)-k_{1}^{2} k_{o} J_{n}\left(k_{1} a_{d}\right) H_{n}^{(2)}\left(k_{o} a_{d}\right)}$,

where $k_{1}$ is the wave number in the dielectric cylinder, and the primes indicate a derivative with respect to the argument [23]. The magnetic currents for both of these scenarios are plotted in Fig. 10, where it is seen that both the amplitude and the phase of the magnetic currents vary across the boundary of the PEC scatterer.

\section{A. Measured results}

Using the same setup described previously, the complex weights of the 12 magnetic-dipole antennas are set using both the voltage-controlled phase shifters and the fixed attenuators. These results will now be included in series with the phase shifters, with the appropriate attenuation given by Fig. 10.

Measuring the fields inside the waveguide, the changes in the scattering around the aluminum cylinder can be monitored when the magnetic currents given in Fig. 10 are imposed on the boundary. Examining first the weights that make the cylinder appear to be a smaller cylinder with a radius of $a_{d}=0.2 \lambda$, the measured fields are plotted in Fig. 11(a). Also plotted are the

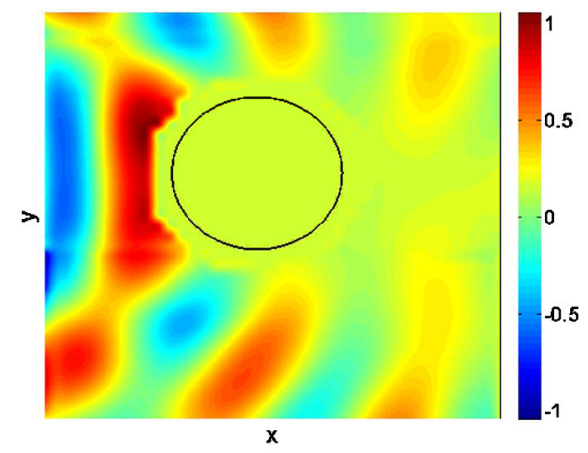

(b)

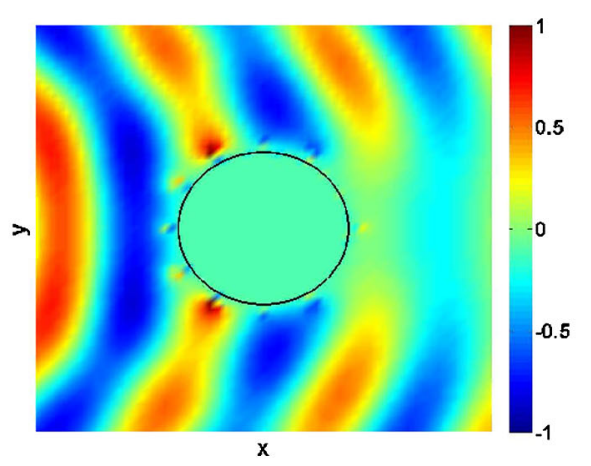

(c)

FIG. 11. (a) The measured real part of the total electric field, with the weights of the magnetic-dipole array set up to disguise the cylinder as a smaller metallic cylinder. Note how the shadow region narrows. (b) The measured real part of the total electric field of the bare aluminum cylinder [repeated from Fig. 4(a) for ease of comparison]. (c) The simulated electric field found using COMSOL for disguising a PEC cylinder as a smaller PEC cylinder. 
full-wave simulation for the same setup, and for convenience, the measured fields of the bare PEC cylinder are shown again. Here, it can be noted that both the measured and simulated results show good agreement. Examining the measured results between the disguised cylinder and the bare cylinder, we see that the main difference between the two is the narrowing of the shadow region around the cylinder. This narrowing is indicative of the smaller appearance of the $0.56 \lambda$ cylinder through the active modification of the scattered field created by the magnetic-dipole array.

For the second example, the measured fields are plotted in Fig. 12, along with the full-wave simulated results of the same setup, as well as a full-wave simulation of the scattering off of a dielectric cylinder with radius $a_{d}=0.4 \lambda$ and $\varepsilon=10$. Here, again, a good agreement between all three plots is seen. By inspecting the shadow region of the cylinder, there is a clear modification of the fields in this region, with the fields now resembling those of the dielectric cylinder shown in Fig. 12.

From these two examples, we show that by having control of the amplitude and phase of the magnetic dipoles that form the cloak, the scattering of the aluminum cylinder can be tailored in a number of ways to disguise an object instead of simply cloaking it.

\section{ESTIMATING THE INCIDENT FIELD}

There are many practical scenarios where the incident field is known a priori, such as when an object is blocking the line of sight of an antenna array [30]. However, in many situations, it is desirable to do cloaking without a priori knowledge of the incident field to determine the weights of the magnetic-dipole array. We may then ask if it is possible to estimate the incident field with as little prior knowledge as possible about the incident field itself. Here, a possible way to accomplish this for a cylindrical PEC object is described. We also note that in Ref. [2], a prescription is given for sensing the field in real time to set the weights for an active cloak. Here, however, a scenario for a steadystate (or frequency-domain) incident field is considered in the presence of a scatterer.

Considering, again, the cloak described above, one can observe that the magnetic-dipole array operates in a transmitting mode of operation to cloak the PEC cylinder from a prescribed incident field. However, the same array, which surrounds the object, can potentially be used as a receiver to detect the incident field scattering off of the PEC object. To examine this detection in more detail, the unknowns surrounding the incident field are first detailed.

We first assume that the cylinder lies in the far field for any source and that the incident field is a plane-wave-like

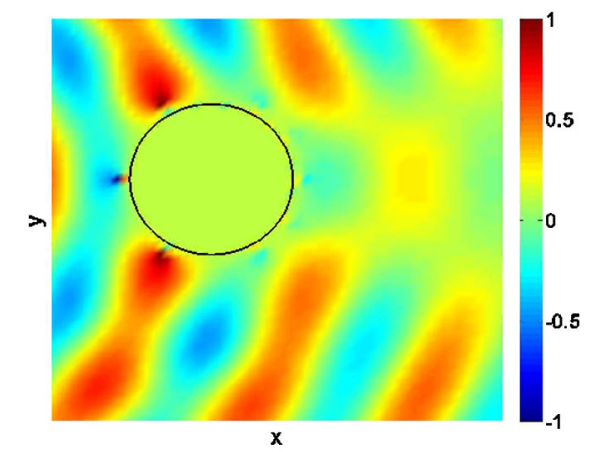

(b)

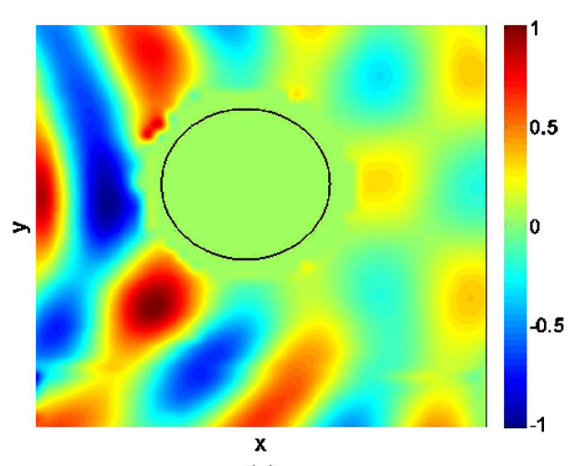

(a)

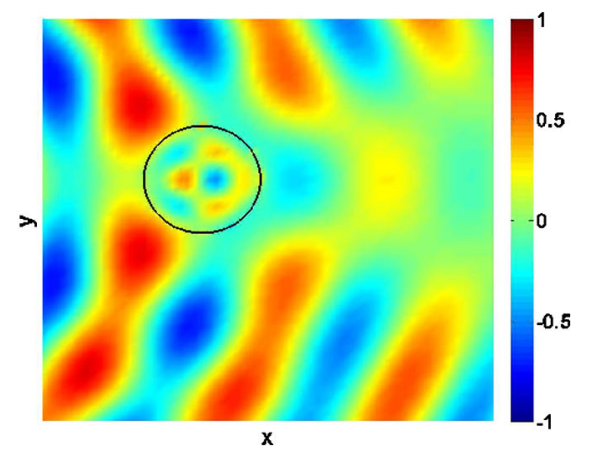

(c)

FIG. 12. (a) The measured real part of the total electric field, with the weights of the magnetic-dipole array set up to disguise the cylinder as a smaller dielectric cylinder. Note how the shadow region has changed. (b) The simulated total electric field found using COMSOL for disguising a PEC cylinder as a smaller dielectric cylinder. Note the good agreement with Fig. 12(a). (c) The simulated total electric field for a dielectric cylinder of radius $r=0.4 \lambda$ and dielectric constant $\varepsilon=10$ found using COMSOL. 
excitation. This is a fair assumption, as for any reasonable scenario, an unknown source would have a plane-wavelike appearance within the vicinity of the object. Because of the narrow-band nature of our setup, we also assume that the frequency is known, though more advanced signal processing techniques may not require this assumption. With this in mind, one can observe that the plane wave, described by $E_{o} \exp \{j(\omega t-\mathbf{k} \cdot \mathbf{r}+\theta)\}$, has three unknowns: the amplitude $E_{o}$, the phase $\theta$, and the direction $\mathbf{k} \cdot \mathbf{r}$. Thus, the signal received by the magnetic-dipole array must allow for these three qualities to be determined.

To accomplish this, knowledge about the object that is being cloaked can also be used advantageously. Here, in this example, the specific characteristics for scattering off of a PEC cylinder can be used. Note, again, that the receiver is a magnetic-dipole array around the boundary of this cylinder. The orientation of this array, as seen in Fig. 3, is such that each loop antenna is excited primarily by the $H_{\varphi}$-field component. (Note that while the boundary condition of the PEC cylinder requires the $E_{z}$ component to be zero, the magnetic field is nonzero at the boundary.) Thus, one can at least predict the general structure of the total $H_{\varphi}$ field (incident plus scattered) that would be received by the antenna at the boundary of the cylinder from general knowledge of how a plane wave scatters off of a PEC cylinder. This structure is given for an arbitrary incident plane wave as

$$
\begin{aligned}
H_{\varphi}= & -\frac{k_{o} \cos \varphi}{j \omega \mu} E_{o} e^{j \theta} e^{j(\omega t-\mathbf{k} \cdot \mathbf{r})} \\
& +E_{o} e^{j \theta} \frac{k_{o}}{j \omega \mu} \sum_{n=-\infty}^{\infty} j^{-n} A_{n} H_{n}^{\prime(2)}\left(k_{o} a\right) e^{(j n \varphi)},
\end{aligned}
$$

where the primes indicate derivatives with respect to the argument. As we will show, one does not need to measure other field components, as information about the incident plane wave is contained in the $H_{\varphi}$ field.

To see how this can be done, the field given in Eq. (14) is first plotted for arbitrary incident field parameters (magnitude, phase, and direction) near the boundary of the PEC cylinder (specifically, $\lambda / 15$ away from the boundary, which is the location of the loop antennas). The direction of the plane wave is examined first. For a simple and symmetric object like a PEC cylinder, the magnitude of the $H_{\varphi}$ field tells us the direction of the incident plane wave due to the strong shadow region created by the object. Thus, at the point which the minimum in the magnitude of the $H_{\varphi}$ field occurs, the corresponding outward surface normal tells us the direction of the incident plane wave. In the plot shown in Fig. 13, since the minimum occurs at $0^{\circ}$, and the outward surface normal points in the $\hat{\mathbf{x}}$ direction, it can be deduced that the incident plane wave travels in that direction. Obviously, for a more complicated object, more complicated signal processing would be needed to deduce
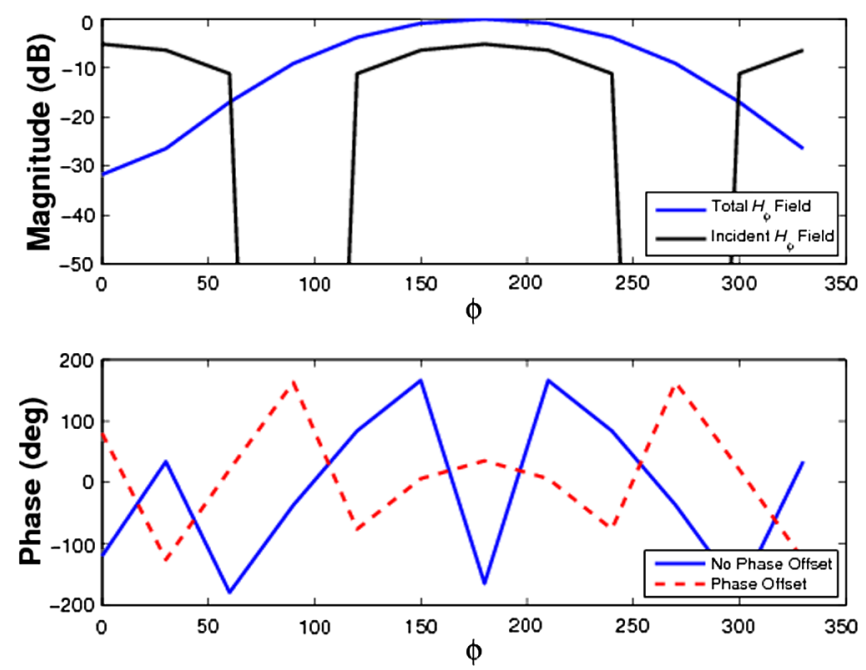

FIG. 13. The tangential magnetic field $H_{\varphi}$ (magnitude and phase) at the boundary of a PEC cylinder when illuminated with a plane wave (blue line). The black line in the upper plot is the magnitude of the magnetic field of the incident plane wave when compared to the magnitude of the total magnetic field at the boundary. The red dashed line in the lower plot is the change in phase for a constant $200^{\circ}$ phase offset.

the direction of arrival [31], but it can be seen that, at least for a symmetrical object, simple assumptions can be made.

To calculate the phase of the incident field, one can simply fit a constant phase offset (with respect to a $0^{\circ}$ reference at the center of the object). This offset is seen in Eq. (14), where the phase of the incident field, $\theta$, is common to all terms. This phase offset then determines the overall phase of the incident field and the corresponding phase of the required magnetic-dipole array. To see how the phase changes with a phase offset, the phase is plotted in Fig. 13 with a phase offset of $\theta=200^{\circ}$.

Finally, the magnitude of the incident plane wave can be compared to the magnitude of the total field, as shown in Fig. 13. Here, we show that given an incident plane wave, the offset between the measured total field and amplitude of the plane wave at broadside is approximately $-5 \mathrm{~dB}$. Thus, by measuring the magnitude of the total $H_{\varphi}$ field at the boundary, information about the magnitude of the incident field can be determined by assuming a planewave incidence. From these simple observations, the key properties of the incident plane wave have been deduced.

The theoretical observed field given by Eq. (14) can be compared with the measured field using the waveguide setup of the previous sections. Here, the (normalized) transmission $\left(S_{21}\right)$ from the monopole antenna to each loop antenna is measured and also plotted in Fig. 14. Note that the general trend in both the magnitude and phase of the received signal at each element of the magnetic-dipole array corresponds well to the predicted theoretical result. Note also that in the measured scenario, there is a point source, not a plane wave because of 

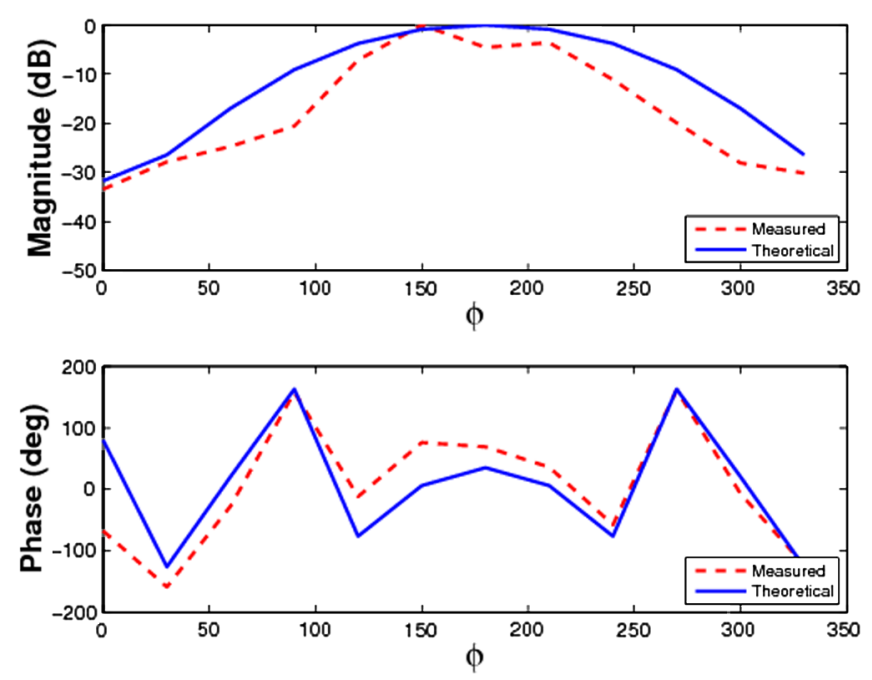

FIG. 14. The theoretical total tangential magnetic field $H_{\varphi}$ at the boundary of a PEC cylinder (blue line) compared to the measured values found using the loop antennas in the waveguide measurement (red dashed line).

limitations with the measurement setup. Thus, from these measured received signals, the properties of the incident field are calculable. A realistic real-time setup of this scenario would use the received signals found using a standard receiver circuitry to calculate the incident field and dynamically set the weights to setup the cloaking effect.

In this example, the specific knowledge about the size, shape, and material composition of the scatterer has been used to predict the incident field and thus the required parameters for the cloak. As discussed in the Introduction, other cloaking schemes have attempted more feasible cloaking mechanisms by making assumptions about the object being cloaked and/or the incident field itself [6-8], and we have shown here how these assumptions can also be useful in the active regime.

As discussed in Ref. [2], causal electromagnetic cloaking is not possible. Thus, determining the parameters of the incident field cannot be accomplished by the magneticdipole array without letting any of the incident field scatter off of the object. However, once the properties of the incident field are found, the appropriate weights on the magnetic-dipole array can be set, and the magnetic-dipole array can conceal the object from the incident field (assuming a steady state). Here, also note that the receive and transmit functions are assumed to be operating at separate times, where the receiver senses the environment and the transmitter responds to it. Hence, this behavior is perhaps better termed as camouflaging instead of cloaking. A simultaneous receiver and transmitter would also have to take into account noise and stability concerns.

What has been shown here is a simple example of how the incident field could be determined by an antenna array that can then be used to hide the object itself. For more arbitrary geometries, more thorough knowledge of how the object scatters the electromagnetic field would be required, perhaps through numerical analysis, and more advanced signal processing of the received signal would be required to determine the magnitude, phase, and direction of the incident field. Another implicit assumption is the narrowband steady-state properties of the incident (and scattered) field. Here again, more advanced signal processing would be required to handle transient, pulsed, or wideband signals.

\section{CONCLUSION}

In this paper, an experimental demonstration of the concept of active interior cloaking for a predetermined electromagnetic field has been presented. Using a formula based on the equivalence principle [19], a cloak for an aluminum cylinder made from a magnetic-dipole array has been constructed. Measuring the cloak in a parallel-plate waveguide demonstrated the cloaking effect, as well as other possible configurations that allow the cylinder to be disguised as another object. Finally, using the magneticdipole array as a receiver, we showed how the incident field could be determined, which could lead to a camouflagingtype behavior.

By using these active sources, the appeal of this approach is that it requires only a thin conformal layer to construct the cloak, using well-known technology from phased-array theory. No artificial materials or surfaces with extreme parameters are required.

Further work on this approach to the problem of cloaking could involve a complete 3D array of electric and magnetic dipoles to cloak dielectric objects, and more sophisticated circuitry to improve the performance of the array. Finally, combining the receive and transmit capabilities of the dipole array to dynamically camouflage an object would be of further interest.

\section{ACKNOWLEDGMENTS}

One of the authors (M.S.) would like to thank Seyed Yasrebi for assisting with the design and measurement of the loop antenna and Liang Liang for helping to physically set up and take down the waveguide. Financial support from the Natural Sciences and Engineering Research Council of Canada (NSERC) is gratefully acknowledged.

[1] D. Schurig, J. J. Mock, B. J. Justice, S. A. Cummer, J. B. Pendry, A.F. Starr, and D. R. Smith, Metamaterial Electromagnetic Cloak at Microwave Frequencies, Science 314, 977 (2006).

[2] David A. B. Miller, On Perfect Cloaking, Opt. Express 14, 12457 (2006).

[3] Steven A. Cummer, Bogdan-Ioan Popa, David Schurig, David R. Smith, and John Pendry, Full-Wave Simulations 
of Electromagnetic Cloaking Structures, Phys. Rev. E 74, 036621 (2006).

[4] Boubacar Kanté, Dylan Germain, and André de Lustrac, Experimental Demonstration of a Nonmagnetic Metamaterial Cloak at Microwave Frequencies, Phys. Rev. B 80, 201104 (2009).

[5] R. Liu, C. Ji, J. J. Mock, J. Y. Chin, T. J. Cui, and D. R. Smith, Broadband Ground-Plane Cloak, Science 323, 366 (2009).

[6] Nathan Landy and David R. Smith, A Full-Parameter Unidirectional Metamaterial Cloak for Microwaves, Nat. Mater. 12, 25 (2012).

[7] Yaroslav Urzhumov, Nathan Landy, Tom Driscoll, Dimitri Basov, and David R. Smith, Thin Low-Loss Dielectric Coatings for Free-Space Cloaking, Opt. Lett. 38, 1606 (2013).

[8] Andrea Alú and Nader Engheta, Plasmonic and Metamaterial Cloaking: Physical Mechanisms and Potentials, J. Opt. A 10, 093002 (2008).

[9] Brian Edwards, Andrea Alù, Mário G. Silveirinha, and Nader Engheta, Experimental Verification of Plasmonic Cloaking at Microwave Frequencies with Metamaterials, Phys. Rev. Lett. 103, 153901 (2009).

[10] D. Rainwater, A. Kerkhoff, K. Melin, J. C Soric, G. Moreno, and A. Alú, Experimental Verification of ThreeDimensional Plasmonic Cloaking in Free-Space, New J. Phys. 14, 013054 (2012).

[11] J. C. Soric, P. Y Chen, A. Kerkhoff, D. Rainwater, K. Melin, and A. Alù, Demonstration of an Ultralow Profile Cloak for Scattering Suppression of a Finite-Length Rod in Free Space, New J. Phys. 15, 033037 (2013).

[12] Sergei Tretyakov, Pekka Alitalo, Olli Luukkonen, and Constantin Simovski, Broadband Electromagnetic Cloaking of Long Cylindrical Objects, Phys. Rev. Lett. 103, 103905 (2009).

[13] Pekka Alitalo, Stanislav Maslovski, and Sergei Tretyakov, Three-Dimensional Isotropic Perfect Lens Based on LC-Loaded Transmission Lines, J. Appl. Phys. 99, 064912 (2006).

[14] P. A. Nelson and S.J. Elliott, Active Control of Sound (Academic Press, New York, 1992).

[15] Radar Handbook, edited by Merrill I. Skolnik (McGrawHill, New York, 1990).

[16] Fernando Guevara Vasquez, Graeme W. Milton, and Daniel Onofrei, Active Exterior Cloaking for the 2D Laplace and Helmholtz Equations, Phys. Rev. Lett. 103, 073901 (2009).
[17] H. H. Zheng, J. J. Xiao, Y. Lai, and C. T. Chan, Exterior Optical Cloaking and Illusions by Using Active Sources: A Boundary Element Perspective, Phys. Rev. B 81, 195116 (2010).

[18] Junjie Du, Shiyang Liu, and Zhifang Lin, Broadband Optical Cloak and Illusion Created by the Low Order Active Sources, Opt. Express 20, 8608 (2012).

[19] Michael Selvanayagam and George V. Eleftheriades, An Active Electromagnetic Cloak Based on the Equivalence Principle, IEEE Antennas Wireless Propag. Lett. 11, 1226 (2012).

[20] Michael Selvanayagam and George V. Eleftheriades, Discontinuous Electromagnetic Fields Using Orthogonal Electric and Magnetic Currents for Wavefront Manipulation, Opt. Express 21, 14409 (2013).

[21] A. E. H. Love, The Integrationg of Equations of Propagation of Electric Waves, Phil. Trans. R. Soc. A 197, 1 (1901).

[22] S.A. Schelkunoff, Some Equivalence Theorems of Electromagnetics and Their Application to Radiation Problems, Bell Syst. Tech. J. 56, 92 (1939).

[23] Roger F. Harrington, Time-Harmonic Electromagnetic Fields (Wiley-Interscience, New York, 2001).

[24] D. Schurig, J. B. Pendry, and D. R. Smith, TransformationDesigned Optical Elements, Opt. Express 15, 14772 (2007).

[25] Stanislav Maslovski, Sergei Tretyakov, and Pekka Alitalo, Near-Field Enhancement and Imaging in Double Planar Polariton-Resonant Structures, J. Appl. Phys. 96, 1293 (2004).

[26] J. Y. Lau and S. V. Hum, Reconfigurable Transmitarray Design Approaches for Beamforming Applications, IEEE Trans. Antennas Propag. 60, 5679 (2012).

[27] Thomas Lee, The Design of CMOS Radio-Frequency Integrated Circuits (Cambridge University, Cambridge, England, 2003).

[28] Walton C. Gibson, The Method of Moments in Electromagnetics (Chapman and Hall/CRC, London, 2008).

[29] John Brown and E. V. Jull, The Prediction of Aerial Radiation Patterns from Near-Field Measurements, Proc. IEEE 108, 635 (1961).

[30] P.-S. Kildal, A. A. Kishk, and A. Tengs, Reduction of Forward Scattering from Cylindrical Objects Using Hard Surfaces, IEEE Trans. Antennas Propag. 44, 1509 (1996).

[31] Joseph Liberti and Theodore S. Rappaport, Smart Antennas for Wireless Communications: IS-95 and Third Generation CDMA Applications (Prentice-Hall, Englewood Cliffs, NJ, 1999). 